\title{
In vivo mapping of functional domains and axonal connectivity in cat visual cortex using magnetic resonance imaging
}

Citation for published version (APA):

Kim, D-S., Kim, M., Ronen, I., Formisano, E., Kim, K-H., Ugurbil, K., Mori, S., \& Goebel, R. (2003). In vivo mapping of functional domains and axonal connectivity in cat visual cortex using magnetic resonance imaging. Magnetic Resonance Imaging, 21(10), 1131-1140. https://doi.org/10.1016/j.mri.2003.08.014

Document status and date:

Published: 01/01/2003

DOI:

10.1016/j.mri.2003.08.014

Document Version:

Publisher's PDF, also known as Version of record

Document license:

Taverne

Please check the document version of this publication:

- A submitted manuscript is the version of the article upon submission and before peer-review. There can be important differences between the submitted version and the official published version of record.

People interested in the research are advised to contact the author for the final version of the publication, or visit the DOI to the publisher's website.

- The final author version and the galley proof are versions of the publication after peer review.

- The final published version features the final layout of the paper including the volume, issue and page numbers.

Link to publication

\footnotetext{
General rights rights.

- You may freely distribute the URL identifying the publication in the public portal. please follow below link for the End User Agreement:

www.umlib.nl/taverne-license

Take down policy

If you believe that this document breaches copyright please contact us at:

repository@maastrichtuniversity.nl

providing details and we will investigate your claim.
}

Copyright and moral rights for the publications made accessible in the public portal are retained by the authors and/or other copyright owners and it is a condition of accessing publications that users recognise and abide by the legal requirements associated with these

- Users may download and print one copy of any publication from the public portal for the purpose of private study or research.

- You may not further distribute the material or use it for any profit-making activity or commercial gain

If the publication is distributed under the terms of Article $25 \mathrm{fa}$ of the Dutch Copyright Act, indicated by the "Taverne" license above, 


\title{
In vivo mapping of functional domains and axonal connectivity in cat visual cortex using magnetic resonance imaging
}

\author{
Dae-Shik Kim ${ }^{\mathrm{a}, *}$, Mina Kim ${ }^{\mathrm{a}}$, Itamar Ronen ${ }^{\mathrm{a}}$, Elia Formisano ${ }^{\mathrm{b}}$, Keun-Ho Kim ${ }^{\mathrm{a}}$, \\ Kamil Ugurbil ${ }^{\mathrm{a}}$, Susumu Mori ${ }^{\mathrm{c}}$, Rainer Goebel ${ }^{\mathrm{b}}$ \\ ${ }^{\mathrm{a}}$ Center for Magnetic Resonance Research, Radiology, University of Minnesota Medical School, Minneapolis, MN, USA \\ ${ }^{\mathrm{b}}$ University of Maastricht, Maastricht, The Netherlands \\ ${ }^{\mathrm{c}}$ Department of Radiology, Johns-Hopkins School of Medicine, Baltimore, MD, USA
}

Received 15 August 2003; received in revised form 22 August 2003; accepted 23 August 2003

\begin{abstract}
Noninvasive cognitive neuroimaging studies based on functional magnetic resonance imaging (fMRI) are of ever-increasing importance for basic and clinical neurosciences. The explanatory power of fMRI could be greatly expanded, however, if the pattern of the neuronal circuitry underlying functional activation could be made visible in an equally noninvasive manner. In this study, blood oxygenation level-dependent (BOLD)-based fMRI and diffusion tensor imaging (DTI) were performed in the same cat visual cortex, and the foci of fMRI activation utilized as seeding points for 3D DTI fiber reconstruction algorithms, thus providing the map of the axonal circuitry underlying visual information processing. The methods developed in this study will lay the foundation for in vivo neuroanatomy and the ability for noninvasive longitudinal studies of brain development. (c) 2003 Elsevier Inc. All rights reserved.
\end{abstract}

Keywords: fMRI; DTI; Visual cortex

\section{Introduction}

Since its introduction in 1992 [1-3], the blood oxygenation level-dependent (BOLD) functional magnetic resonance imaging (fMRI) has revolutionized cognitive neurosciences by allowing the foci of cortical "activity" to be visualized in vivo in a noninvasive manner. The BOLD contrast originates from the intravoxel magnetic field inhomogeneity induced by paramagnetic deoxyhemoglobin $(\mathrm{deoxyHb})$ sequestered in red blood cells that are compartmentalized within blood vessels. The magnetic susceptibility differences between the deoxyHb-containing compartments and the surrounding space generate magnetic field gradients around the boundaries of these compartments. Therefore, perturbation of regional deoxyHb content alters the signal intensities in MR images sensitized to BOLD contrast. Such regional perturbation occurs as the result of

\footnotetext{
* Corresponding author. Current address: Department of Anatomy and Neurobiology, Boston University School of Medicine, Boston, MA, USA. Tel.: +1-617-638-4200; fax: +1-617-638-4216.

E-mail address: dskim@bu.edu (D.-S. Kim).
}

enhanced neuronal activity and metabolism during sensory [4], motor [5], or cognitive [6] functions.

While functional neuroimaging based on BOLD contrast provides detailed information about the "where" of the brain's functional architecture noninvasively, such localization information alone, however, must leave pivotal questions about the brain's information processing (the "how" of the processing) unanswered, as long as the underlying pattern of neuronal connectivity cannot be mapped in an equally noninvasive manner. The explanatory power of the current functional MRI studies could be greatly expanded, therefore, if the same MRI framework could be utilized to assess the pattern of neuronal connectivity in vivo. The newly developed magnetic resonance imaging technique known as diffusion-tensor-imaging (DTI) based on diffusion weighted imaging (DWI) has the potential to serve as such a method.

DWI MRI is one of the most widely used MRI methods for investigating the microscopic structure of water-containing material in general, and living tissue in particular [7]. As suggested by Stjeskal and Tanner [8], the MR image is sensitized to diffusion in a given direction using a couple of temporally separated magnetic field gradients in the desired 
direction. The first gradient labels the spin phase of the water molecule protons along the gradient direction, while the second gradient rephrases the spin phase. If no diffusion has occurred during the time period between the two gradients, the initial phase is fully restored, while the initial phase is not fully recovered if water molecule displacement has taken place. In such a case, the resulting image intensity is attenuated. The amount of image attenuation, or "diffusion weighting," is given by the relation $S(b)=S_{0}$ $\exp \left(-b_{i} D_{i}\right)$ where $\mathrm{S}$ is the signal intensity and $\mathrm{S}_{0}$ is the signal intensity without diffusion weighting. $D_{i}$ is the diffusion coefficient of water in the direction on which of the magnetic field gradient was applied, and $b$ is given by: $b=$ $\gamma^{2} g^{2} \delta^{2}(\Delta-\delta / 3)$ in a spin-echo experiment, where $g$ is the gradient strength, $\gamma$ is the gyromagnetic ratio of protons, $\delta$ is the gradient duration time, and $\Delta$ is the gradient separation time.

Because the parameters $g, \gamma, \delta$, and $\Delta$ are all known, from the amount of signal decrease $\left(S / S_{0}\right)$, diffusion constants at each voxel can be derived. Such measurements have revealed that diffusion of brain water has strong directionality (anisotropy), which is attributed to the existence of natural boundaries, such as axons and/or myelination. The properties of such water diffusion can be expressed as an ellipsoid [9]. Such "diffusion ellipsoids" can be characterized by six parameters: diffusion constants along the longest, middle, and shortest axes $\left(\lambda_{1}, \lambda_{2}\right.$, and $\lambda_{3}$, called principal axes); and the direction of the three principal axes. Once the diffusion ellipsoid is fully characterized at each pixel of the brain images, local fiber structure can be derived. For example, if $\lambda_{1} \gg \lambda_{2} \geq \lambda_{3}$ (diffusion is anisotropic), it suggests the existence of dense and aligned fibers within each pixel, whereas isotropic diffusion $\left(\lambda_{1} \approx \lambda_{2} \approx\right.$ $\lambda_{3}$ ) suggests sparse or unaligned fibers.

If one assumes that the orientation of the axis associated with $\lambda_{1}$ is co-linear with the direction of the axonal fibers dominant within the imaging voxel, then DTI can reconstruct the presumed fiber trajectories from a discrete set of diffusion tensors [9,10]. Mori et al. [11] developed one of the earliest and most commonly employed algorithms for DTI-based 3D fiber tracking (called FACT). This scheme is based on extrapolation of continuous vector lines from discrete DTI data. The reconstructed fiber direction within each voxel is parallel to the diffusion tensor eigenvector associated with the greatest eigenvalue $\left(\lambda_{1}\right)$. Within each voxel the fiber tract is a line segment defined by the input position, the direction of the eigenvector, and an output position at the boundary with the next voxel. The track is propagated from voxel to voxel. Tracking is terminated when a sharp turn in the fiber orientation occurs. In algorithms developed by Basser et al. [10], Conturo et al. [12], and Pajevic [13] a continuous representation of the diffusion tensor and principal eigenvector are interpolated from the discrete voxel data. The fiber track direction at any location along the track is given by the continuous eigenvector. Typically, the tracking al- gorithm stops when the fiber radius of curvature or the anisotropy factor falls below a threshold.

In the past few years, numerous other DTI fiber reconstruction algorithms have been proposed that reveal spectacular images of axonal connectivity patterns in vivo in humans $[10,12,14]$, rodents $[9,15]$, and cats [16]. The differences in detailed fiber reconstruction algorithms notwithstanding, all DTI-based fiber reconstructions are faced with a fundamental and peculiar problem: unlike conventional neurotracing techniques (e.g., using DiI, HRP, biocytin etc.; see Discussion), each DTI experiment has the potential to provide the complete set of connectivity across all imaged voxels. Consequently, if the fiber reconstructions are not limited to particular regions of interest (ROI), the resulting pattern of fiber connections would be rendered useless. A key in making DTI an outstanding technique for cognitive neurosciences is therefore to develop selection criteria to determine the seeding region of interest (ROI) for DTI fiber tracing. To this end, in the majority of fiber-tracking algorithms, tracking starts at a user-defined seeding point or region of interest. Such "seeding points" are selected either based on the quality of the underlying DWI, or based on a priori anatomic criteria that are known from postmortem studies. Such anatomically motivated tracking strategies are of greatest importance for testing for anatomic irregularities in vivo. However, in normal subjects, strictly anatomically defined DTI fiber reconstructions have the tendency to simply "rediscover" what has been known from conventional anatomic and histologic techniques, thus resulting in partially tautological statements.

An alternative way of DTI fiber reconstruction is to use the foci of functional activity-such as obtained with BOLD contrast-as the "initial" and "termination" ROIs. This is a more natural choice for most questions in cognitive neurosciences, as the main interest here is to elucidate the pattern of neuronal circuitry underlying the observed functional activation for a particular task.

In this study, BOLD-based fMRI and DTI were performed in the same cat visual cortex at 9.4 Tesla magnetic fields, and the foci of fMRI activation utilized as seeding points for 3D DTI fiber reconstruction algorithms, thus providing the map of the axonal circuitry underlying visual information processing. The methods developed in this study will lay foundation for in vivo neuroanatomy and the ability for non-invasive longitudinal studies of brain development.

\section{Materials and methods}

\subsection{Animal preparation}

Juvenile cats were treated with atropine sulfate $(0.05$ $\mathrm{mg} / \mathrm{kg}$, subcutaneous (s.c.)) and initially anesthetized with a ketamine/xylazine cocktail (10-25 mg/kg ketamine hydrochloride; $2.5 \mathrm{mg} / \mathrm{kg}$ xylazine hydrochloride; both intramus- 


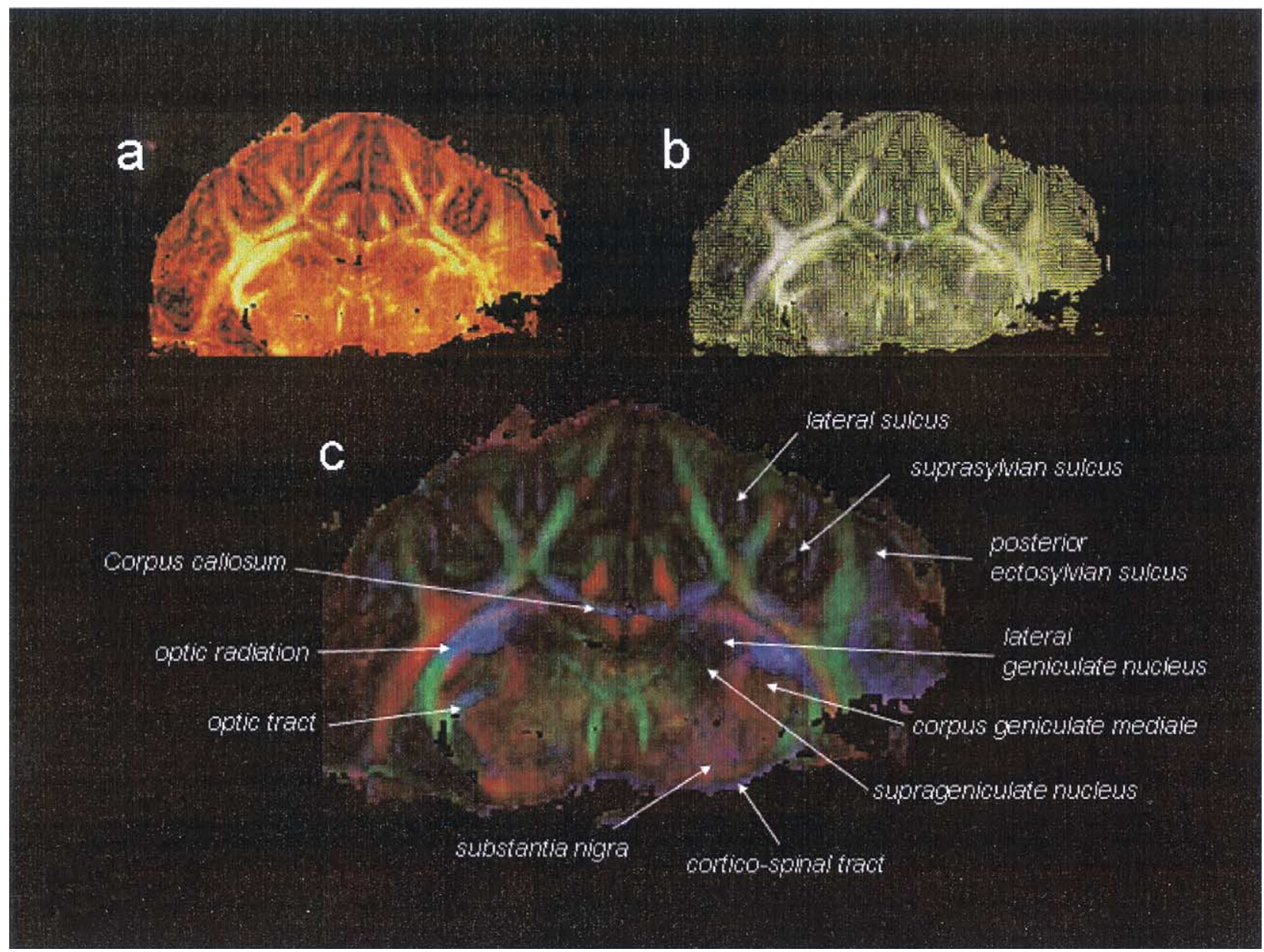

Fig. 1. High-resolution diffusion tensor imaging (DTI) in cat visual cortex. All images are from the same coronal slice around anterior-posterior 0 of the cat cortex (i.e., primary visual cortex). a, the anisotrophy maps in which the FA were color-coded on a voxel-by-voxel basis (bright, high FA; dark, low FA). Note that the regions of the highest fractional anisotrophy are confined to areas of white matter consisting of large fiber bundles. $\mathrm{b}$ and $\mathrm{c}$, vector and color representations of the local anisotrophy, respectively. In c, fibers running in medio-lateral direction are coded in blue, and fibers running in ventro-dorsal directions are coded in green. Areas coded in red correspond to fibers that are running orthogonal to the imaging plane (i.e., they are running in anterior-posterior direction).

cular). A venous catheter was placed in one of the forearm veins (V. cephalica antebrachii), a urinary catheter was placed, and a tracheal tube was inserted for artificial ventilation. Expiratory $\mathrm{CO}_{2}$ content was monitored continuously by a capnometer (Datex Ohmeda) and kept at about 3.5\% by varying ventilation volume and frequency. Gaseous anesthesia (isoflurane $(0.6-1.5 \%)$ in a mixture of $70 \%$ nitrous oxide and $30 \%$ oxygen) was induced and maintained through the scan. The eyes were refracted, and corrective contact lenses placed if necessary. Animals were paralyzed with pancronium bromide (dose $0.1 \mathrm{mg} / \mathrm{kg}$ i.v. every 30 min). The animal's head position was fixed by a mouth-bar and head-holder. A quadrature surface RF coil was placed above area 18 (AP3 on Horsley-Clark coordinate) of the cat visual cortex. Visual stimuli were generated using the VSG (Cambridge Research Systems, UK), and displayed on a rear-projection screen using a video projector (NEC; reso- lution $1040 \times 890$ ). The rear-projection screen was positioned $15 \mathrm{~cm}$ from the animal's eyes, covering about $37^{\circ}$ of the visual field. Stimuli were presented binocularly. Conventional retinotopic stimuli were employed.

\subsection{MRI data acquisition methods}

MR experiments were performed on a 9.4-T/31-cm horizontal MRI scanner (Magnex, UK) equipped with a Unity INOVA console (Varian, CA), and a 30-G/cm actively shielded gradient insert (ID $=11 \mathrm{~cm}$ ) capable of $300-\mu \mathrm{s}$ risetime (Magnex, UK). Magnetic field homogeneity was optimized using automatic shimming routines (FASTMAP). The MR parameters for $\mathrm{T}_{2}{ }^{*}$-based BOLD functional MRI were: gradient-echo echo-planar imaging (GRE-EPI) with field of view of $3.4 \times 3.5 \times 2.0 \mathrm{~cm}^{3}$ and $64 \times 64 \times 13$ data matrix; TE $=14 \mathrm{msec}$, image TR $=1.5 \mathrm{sec}$. For diffusion- 


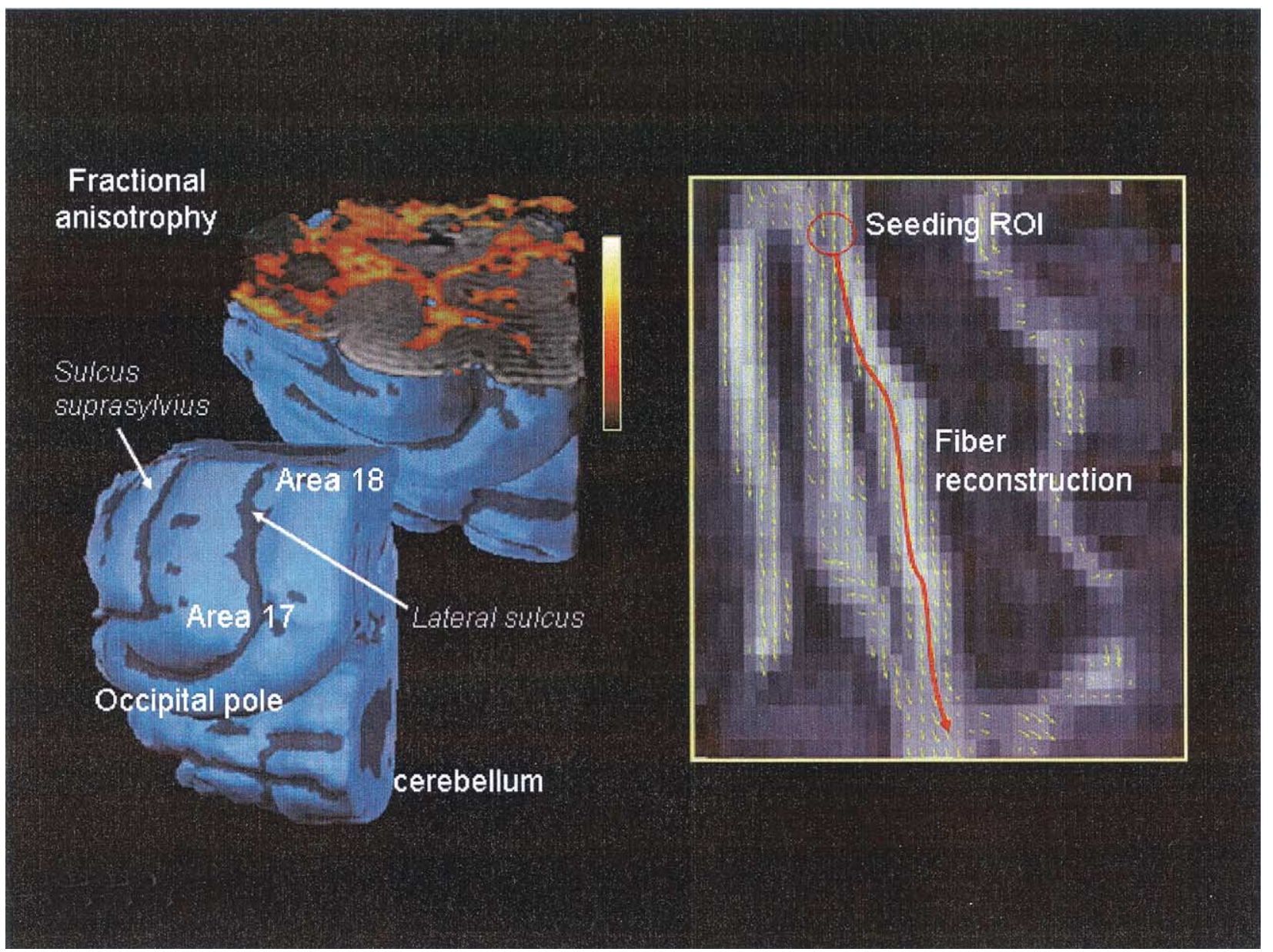

Fig. 2. Lower left, 3-D rendering of the cat cortex around the occipital pole. The gyri and sulci of are displayed in bright and dark blue colors, respectively. Upper left, resliced view of the same rendered cortex. Here, the corresponding FA map from the DTI experiment was projected onto a coronal slice. The regions of largest FA are confined to the white matter areas consisting of large fiber bundles. Right, vector representation of anisotrophy from the same cortex.

weighted MRI, a 3D double spin-echo echo-planar imaging (SE-EPI) sequence with four segments was used. DW MR images were acquired using the following parameters; $\mathrm{g}=$ 17 gauss $/ \mathrm{cm}, \delta=0.006 \mathrm{~s}, \Delta=0.015 \mathrm{~s}\left(\mathrm{~b}=1584 \mathrm{~s} / \mathrm{mm}^{2}\right.$ for each direction). The field of view (FOV) was $5 \times 5 \times 3 \mathrm{~cm}^{3}$, the matrix size was $128 \times 128 \times 64$. The gradient scheme used for the present study is given in the following table:

\begin{tabular}{rrr}
\hline $\mathrm{G}_{\mathrm{x}}$ & $\mathrm{G}_{\mathrm{y}}$ & $\mathrm{G}_{\mathrm{z}}$ \\
\hline 1 & 1 & 0 \\
1 & 0 & 1 \\
0 & 1 & 1 \\
-1 & 0 & 1 \\
1 & -1 & 0 \\
0 & 1 & -1 \\
0 & 0 & 0 \\
\hline
\end{tabular}

Here, $G_{x}, G_{y}, G_{z}$ code for the applied gradient in readout, phase-encoding, and slice selection directions, respectively. The numbers " 1 ," "- 1 ," and " 0 " stand for the presence of positive, negative, and no gradients, respectively.

\subsection{Diffusion tensor calculation}

Diffusion tensor elements were calculated using the following set of equations. Here, the letters $\mathrm{X}, \mathrm{Y}$, and $\mathrm{Z}$ in parentheses denote the directions along which the gradients were applied, and the subscript of $\mathrm{D}$ denotes the respective element of the diffusion tensor $\overline{\bar{D}}, S_{0}$ is the image without diffusion weighting, and $S_{x y z}$ are the diffusion-weighted images where the gradients are applied along the direction defined in the subscript:

$$
\begin{aligned}
& (\mathrm{X}, \mathrm{Y}, 0):\left(D_{x x}+D_{y y}+2 D_{x y}\right)=-\frac{\ln S_{110} / S_{0}}{b} \\
& (\mathrm{X},-\mathrm{Y}, 0):\left(D_{x x}+D_{y y}-2 D_{x y}\right)=-\frac{\ln S_{1-10} / S_{0}}{b}
\end{aligned}
$$

$$
(\mathrm{X}, 0, \mathrm{Z}):\left(D_{x x}+D_{z z}+2 D_{x z}\right)=-\frac{\ln S_{101} / S_{0}}{b}
$$




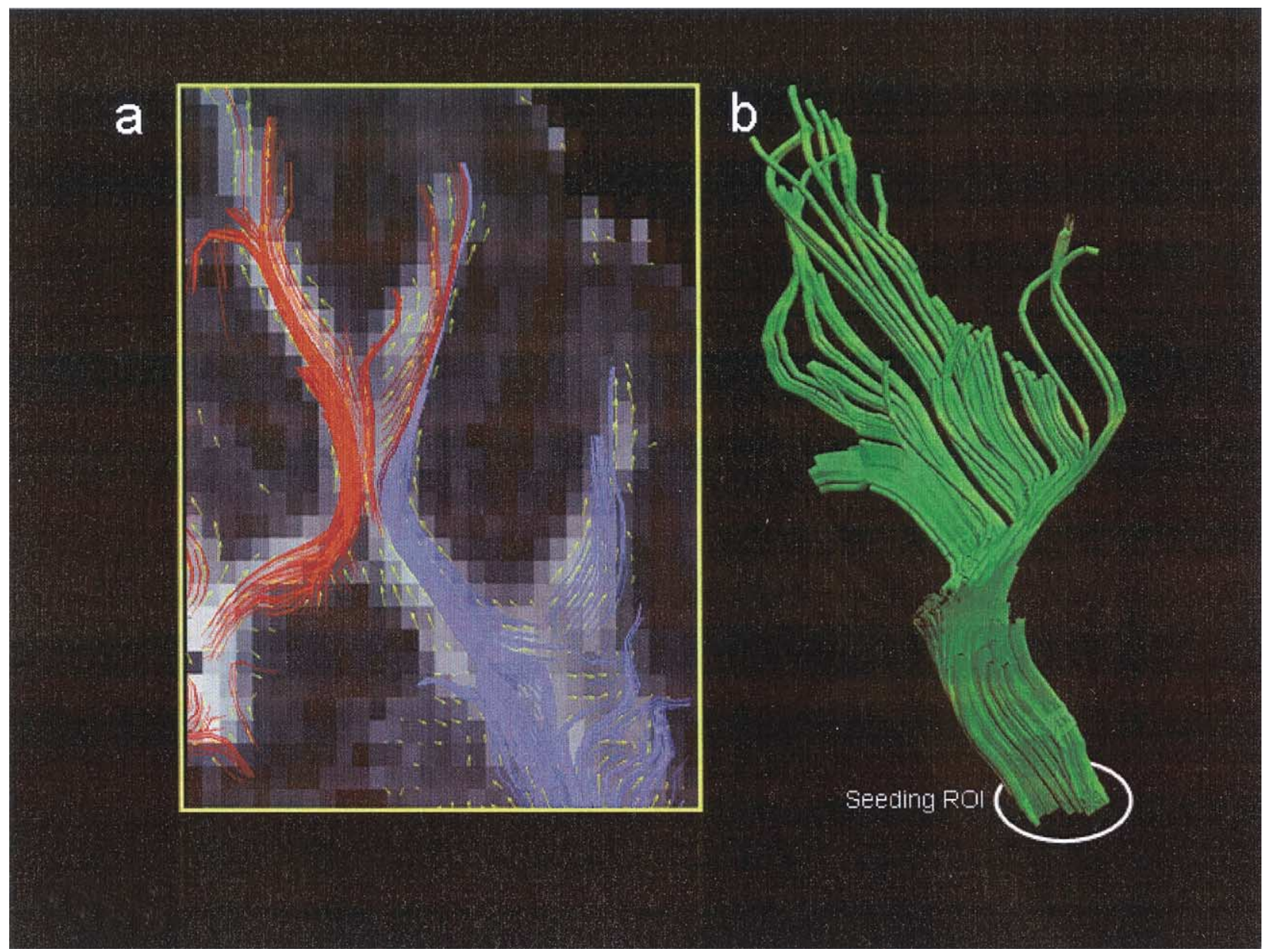

Fig. 3. Left, 2-D projection of fiber reconstructions. The fibers originating from two seeding ROIs are displayed in colors red and blue, respectively. Coronal slice of the cat primary visual cortex. Right, the result fiber reconstruction as original 3-D trajectories.

$$
\begin{aligned}
& (-\mathrm{X}, 0, \mathrm{Z}):\left(D_{x x}+D_{z z}-2 D_{x z}\right)=-\frac{\ln S_{-101} / S_{0}}{b} \\
& (1-4) \\
& (0, \mathrm{Y}, \mathrm{Z}):\left(D_{y y}+D_{z z}+2 D_{y z}\right)=-\frac{\ln S_{011} / S_{0}}{b} \\
& (0, \mathrm{Y},-\mathrm{Z}):\left(D_{y y}+D_{z z}-2 D_{y z}\right)=-\frac{\ln S_{01-1} / S_{0}}{b}
\end{aligned}
$$

From the equations (1-1)- (1-6), we can form a tensor matrix [20]:

$$
\overline{\bar{D}}=\left[\begin{array}{lll}
D_{x x} & D_{x y} & D_{x z} \\
D_{x y} & D_{y y} & D_{y z} \\
D_{x z} & D_{y z} & D_{z z}
\end{array}\right]
$$

Since the tensor matrix $\overline{\bar{D}}$ is symmetric along the diagonal, the eigenvalues and eigenvectors can be obtained by diagonalizing the matrix using the Jacobi transformation. The resulting eigenvalues $\overline{\bar{\Lambda}}=\left[\begin{array}{lll}\lambda_{1} & 0 & 0 \\ 0 & \lambda_{2} & 0 \\ 0 & 0 & \lambda_{3}\end{array}\right]$ and corresponding eigenvectors $\overline{\bar{P}}=\left[\overrightarrow{p_{1}} \overrightarrow{p_{2}} \vec{p}_{3}\right]$ can then be used to describe the directionality of water diffusion within a given voxel. There are several different methods for how this can be achieved. For the present study, the directionality of water diffusion was estimated by computing the fractional anisotropy (FA) on a voxel-by-voxel basis [17].

$$
\begin{aligned}
F A & =\frac{1}{\sqrt{2}} \times \\
& \sqrt{\frac{\left(\lambda_{1}-\lambda_{2}\right)^{2}+\left(\lambda_{2}-\lambda_{3}\right)^{2}+\left(\lambda_{3}-\lambda_{1}\right)^{2}}{\lambda_{1}{ }^{2}+\lambda_{2}{ }^{2}+\lambda_{3}{ }^{2}}}
\end{aligned}
$$

The fractional anisotropy (FA) is a measure of the directionality of the water diffusion within a given voxel. 


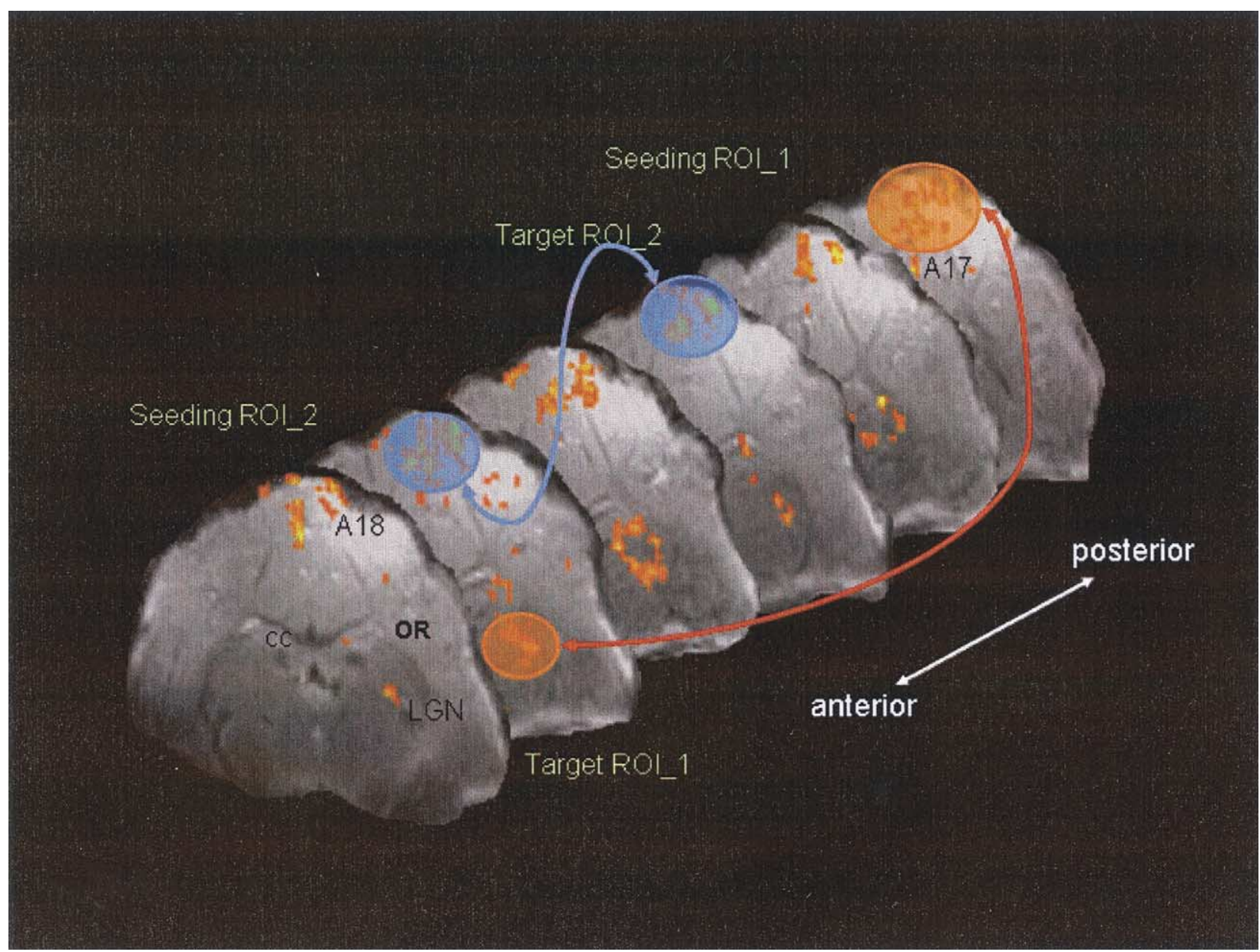

Fig. 4. A series of coronal slices covering roughly the anterior-posterior extend of the cat primary visual cortex. The fMRI time series obtained during the stimulation of the animal visual retinotopic stimuli were used to label the areas of high BOLD activity (in yellow-orange scale). Functional activity was most pronounced in the visual areas 17, 18, and around the thalamic nuclei LGN (lateral geniculate nucleus). Seeding ROIs for DTI based fiber reconstructions were places within the BOLD activation areas.

\subsection{DTI fiber reconstruction}

Based on the diffusion tensors, a fiber-tracking algorithm was applied to the data using custom-written $\mathrm{C}++$ or Matlab (Mathworks, MA) codes. The algorithm of choice was one based on the Frenet equation, similar to Basser's algorithm [10], additionally modified to include a criterion for the vector field interpolation in order to remove noise and background effects (see [18] for details). The FA threshold for stopping the tracking procedure was set to 0.2 . ROIs for seeding the tracking algorithm were chosen either manually or based on BOLD fMRI activation foci. In either case, the seeding ROIs were chosen close to the fiber termini, in order to ensure that they included white matter areas.

\subsection{Functional image construction}

BrainVoyager (Brain Innovation, The Netherlands) was used for functional image construction and projection into the flattened anatomic cortical map. After preprocessing (mean intensity adjustment, interscan slice time-correction,
3D motion correction), functional time-series were co-registered to 3D anatomic images. 3D anatomic images were used for cortical surface reconstruction, inflation, and flattening [19]. Overall, conventional methods of statistical parametric mapping (cross-correlation, GLM etc.) were used for functional image construction. Subsequently, custom-written $\mathrm{C}^{2+}$ and MATLAB routine were used to coregister DTI fiber reconstructions with either fMRI raw data or with inflated/flatted anatomic images. To this end, the areas of high BOLD activity (cross-correlation threshold above 0.1 ; cluster size, 4 voxels) were imported in DWI data as binary masks on which the fiber reconstruction seeding points were then placed.

\section{Results}

Fig. 1 displays the results of high-resolution DTI studies in cat visual cortex obtained at 9.4 Tesla magnetic fields. All images are from the same coronal slice around anteriorposterior 0 of the cat cortex (i.e., primary visual cortex). 


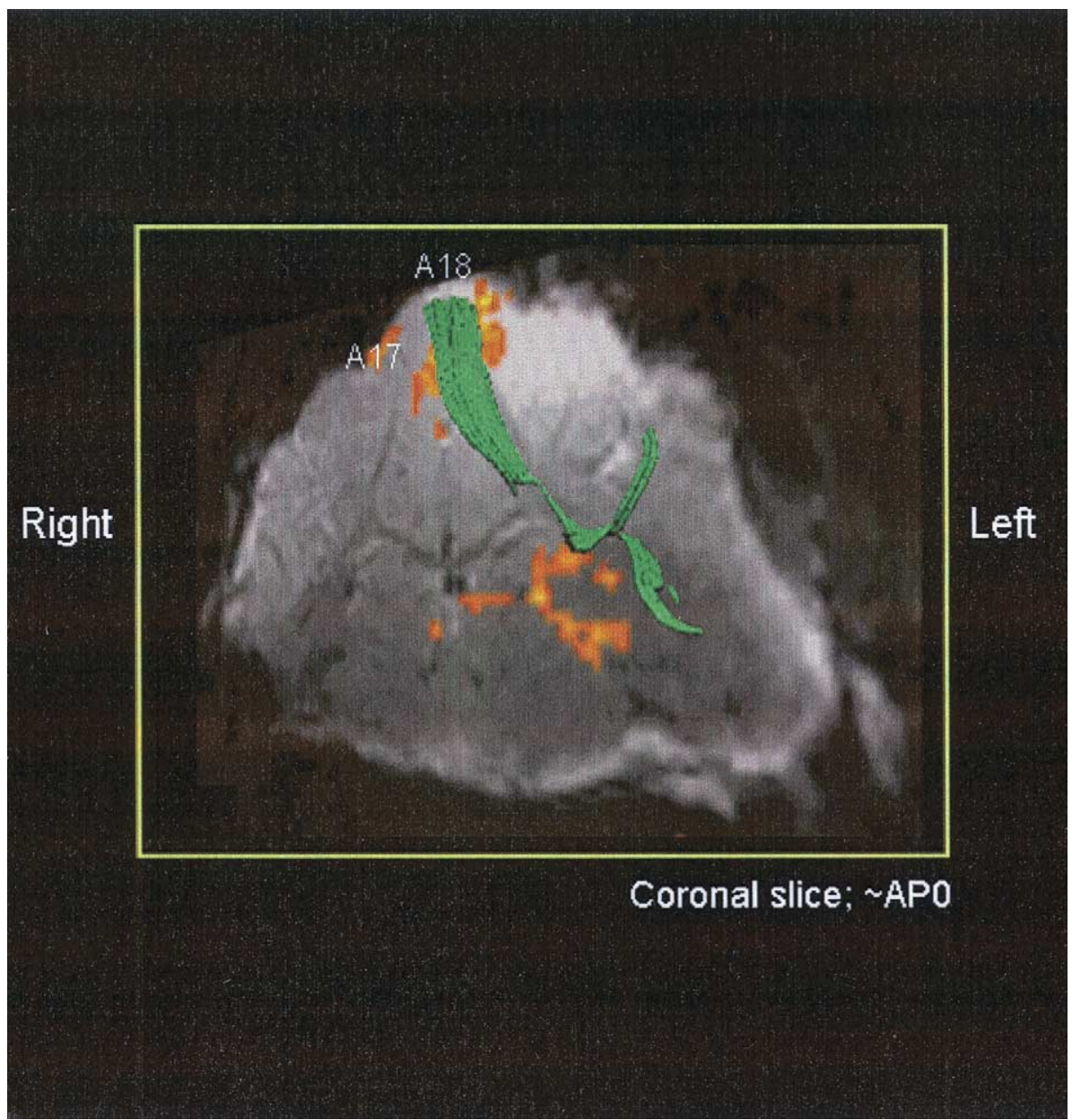

Fig. 5. BOLD-based DTI fiber reconstructions. In this coronal slice, the foci of fMRI activation within areas 17, 18, and LGN were utilized as seeding points for 3D DTI fiber reconstruction algorithms. The resulting fiber reconstruction nicely demonstrates the pattern of connectivity originating from the primary visual areas and LGN, respectively.

Panel a depicts the anisotrophy maps in which the FA were color-coded on a voxel-by-voxel basis (bright, high FA; dark, low FA). Note that the regions of the highest fractional anisotropy are confined to areas of white matter consisting of large fiber bundles. The diagonalized tensor information $\overline{\bar{P}}$ can be represented as a vector. Fig. 1b displays such a vector field map from the cat primary visual cortex. In Fig. $1 \mathrm{c}$, the anatomic details of the cat cortex can be identified in this color-coded map of anisotropy. Here, fibers running in medio-lateral direction are coded in blue, and fibers running in ventro-dorsal directions are coded in green. Areas coded in red correspond to fibers that are running orthogonal to the imaging plane (i.e., they are running in anterior-posterior direction). Note that the areas of large fiber bundles (e.g., running ventro-dorsally into the gray matter) are characterized by bright colors (i.e., high FA) while this is not the case for voxels located within cortical gray matter and/or around the thalamic nuclei.

Fig. 2 shows the strategies employed for the DTI fiber reconstruction. The left panel of Fig. 2 depicts the 3D rendering of the cat cortex around the occipital pole. Here, the gyri and sulci of the are displayed in bright and dark blue colors, respectively. The primary visual areas 17 and 18 of the cat cortex can be identified through their vicinity to the lateral and suprasylvian sulci. The upper left panel of Fig. 2 also shows the resliced view of the same rendered cortex. Here, the corresponding FA map from the DTI experiment (performed during the same MRI study) isafter thresholding - projected onto a coronal slice. The regions of largest FA are confined to the white matter areas consisting of large fiber bundles. The right panel of Fig. 2 shows the vector representation from the same cortex.

For fiber reconstructions, the individual vectors (as displayed in the right panel of Fig. 2) were, starting from a seeding point, concatenated to yield a continuous progress line as described in the method section above. Fig. 3a displays the results of two of such fiber reconstructions. Here, the fibers originating from two seeding ROIs are displayed in colors red and blue, respectively. The fiber trajectories were originally reconstructed in the full 3-D space of the cortex frame. For the display in panel a of Fig. 3 , the 3-D fibers were projected onto the 2-D plane of the displayed coronal slice. Fig.3b shows the 3-D trajectory for one of the two fiber bundles depicted in Fig. 3a. 


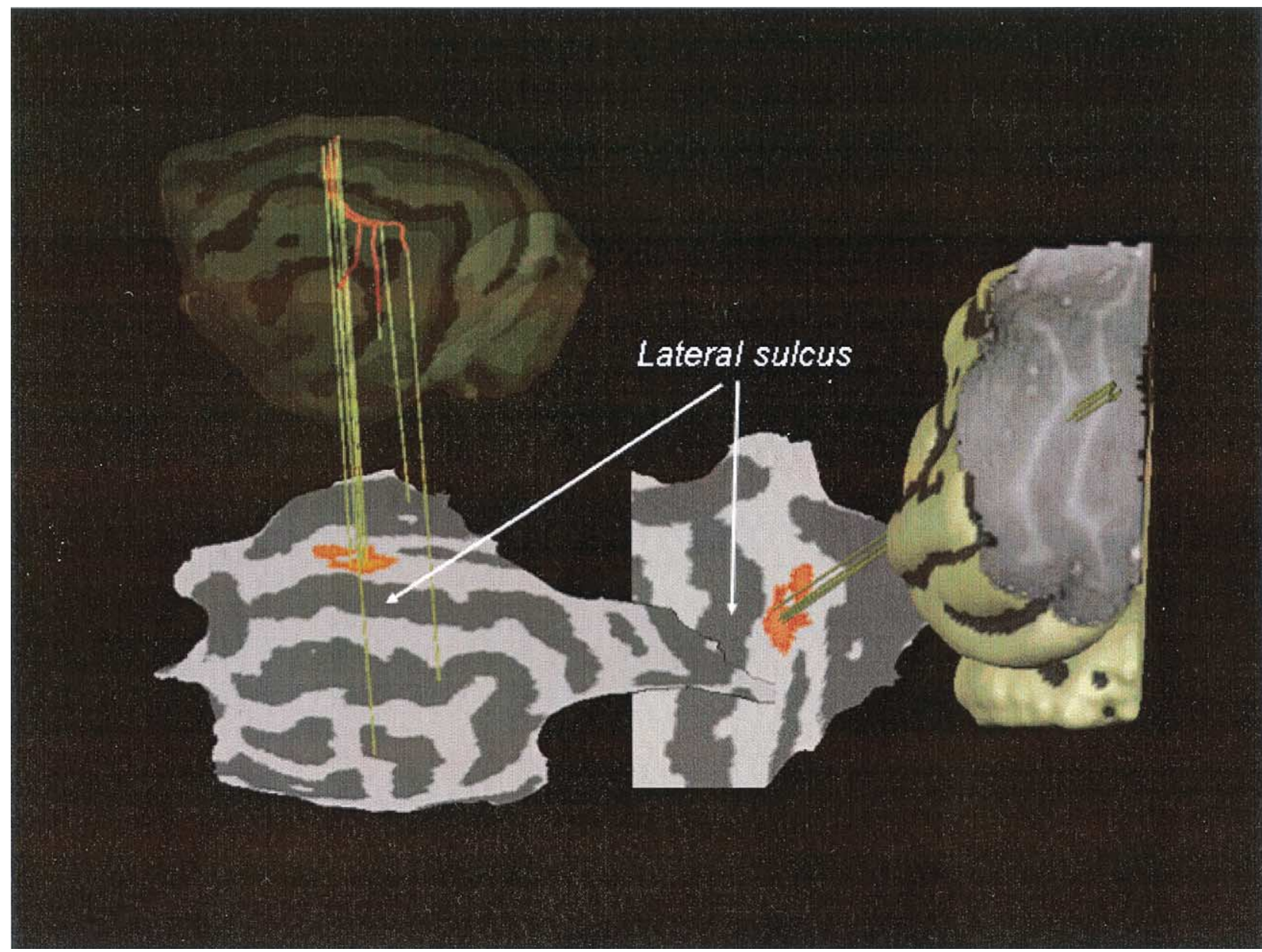

Fig. 6. Upper right, 3-D volume reconstruction of the cat cortex as a translucent "glass brain" in which the BOLD-based DTI fibers were embedded, thus preserving the true 3-D trajectories of the reconstructed fibers. Lower right, "flattened" representation of cortex. Light and dark gray areas code for gyri and sulci, respectively. Corresponding points between reconstructed and flattened cortices were connected through the reconstructed DTI fibers. Fibers coded in red are the fibers that are embedded within the 3-D reconstruction of the cortex. Fibers between points in the flattened cortex representation are coded in yellow. Right, same data as in the left panel viewed from a different perspective.

Fig. 4 shows a series of coronal slices covering roughly the anterior-posterior extend of the cat primary visual cortex. The fMRI time series obtained during the stimulation of the animal with retinotopic stimuli (e.g., expanding checkerboard) were used to label the areas of high BOLD activity (in yellow-orange scale). Functional activity were most pronounced in the visual areas 17, 18, and around the visual thalamic nucleus LGN (lateral geniculate nucleus).

As described in the introduction, in most fiber-tracking algorithms, tracking starts at a user-defined seeding point or region of interest. Alternatively, the "seeding" and "termination" ROIs for DTI fiber tracings can be placed on the areas of high BOLD contrast, as displayed in Fig. 4. The result of one of such BOLD-based DTI fiber reconstructions is shown in Fig. 5. In this coronal slice (around the center of the cat primary visual cortex), the foci of fMRI activation within areas 17, 18, and LGN were utilized as seeding points for 3-D DTI fiber reconstruction algorithms. The resulting fiber reconstruction nicely demonstrates the pattern of connectivity originating from the primary visual areas and LGN, respectively.
In Fig. 5, the fiber trajectories were projected on the 2-D coronal slice. However, a more comprehensive BOLDbased DTI fiber reconstruction will need to take into account the three-dimensional geometry of the cortex. This is a nontrivial task, as the cortex is folded in a complex topology consisting of convex gyri that are visible from outside, and concave sulci that are buried inside. To this end, we have reconstructed the full 3-D volume of the cat cortex as an translucent "glass brain" in which the BOLDbased DTI fibers were embedded, thus preserving the true 3-D trajectories of the reconstructed fibers. Fig. 6 shows such an example. In addition, the reconstructed cortex was "inflated" and subsequently "flattened" using standard methods [19]. The corresponding points between the "glass" and flattened versions of the cortex were then connected through the reconstructed DTI fibers in a topology preserving manner. In Fig. 6, fibers coded in red are the fibers that are embedded within the 3-D volume of the cortex. Fibers between points in the flattened cortex representation are coded in yellow. The right panel of Fig. 6 
shows the same data from a different view angle "through" the reconstructed 3-D cortex.

\section{Discussion}

The results of our study suggest that high-resolution BOLD MRI and DTI can be obtained from the same cortical tissue in vivo at 9.4 Tesla magnetic fields. Furthermore, in our study, the foci of fMRI activation were successfully utilized as seeding points for 3-D DTI fiber reconstruction algorithms, thus providing the map of the axonal circuitry between neuronal populations participating in a common cortical information processing.

Traditionally, connectivities between individual neurons or groups of neurons have been studied using a variety of techniques. These include post mortem methods, such as dissection of white matter, strychnine neuronography [20], and the Nauta [21] methods of tracing degeneration after localized lesions. More recently, implantation of carbocyanine dyes, such as DiI and DiA [22], has replaced the older degeneration methods. However, besides the long duration needed for passive labeling of axonal fibers (often several months), post mortem methods inherently fail to yield a correlation to the foci of functional activation. There are other modern in vivo labeling methods that can be used to track patterns of neuronal connectivity, including transneuronal markers, such as horseradish peroxidase (HRP), rhodamine- and fluorescein-conjugated latex microspheres [23], biocytin [24], and biotinylated dextran amine (BDA). However, all current postmortem and in vivo tracing techniques suffer from common limitations: 1 ) an extremely low number of labeled tracts can be identified; 2) short tracing distance (across 1-3 synapses); 3) the need for invasive injection of the tracer, and most importantly, 4) the need to sacrifice the animal before the labeling pattern can be visualized. Such limitations rule out the use of these methods for human and/or longitudinal studies.

An alternative method to label axonal connectivity in vivo has been recently proposed [25]. In this method, manganese ion $\mathrm{Mn}^{2+}$ is used as a MRI-sensitive in vivo fiber tract tracer. However, while $\mathrm{Mn}^{2+}$ acts as an excellent MRI contrast agent through shortening of tissue $\mathrm{T}_{1}$ ("spin-lattice relaxation"), at the same time it is a potent calcium analog, and topical application leads to epileptic discharge of neuronal activity, contaminating the stimulus induced pattern of cortical activity. Furthermore, $\mathrm{Mn}^{2+}$ is paramagnetic. As such, the accumulation of $\mathrm{Mn}^{2+}$ in cell somata, dendrites, and axons will attenuate and/or corrupt the quality of $\mathrm{T}_{2}{ }^{*}$ weighted images used for BOLD fMRI studies. These not only prohibit the use of $\mathrm{Mn}^{2+}$ for studying neural connections in humans, but it also invalidates its use in animal systems where simultaneous assessment of the functional activity (e.g., BOLD-based) is required.

The BOLD-based DTI fiber reconstruction method described in this study allows the local orientation of fiber bundles in the white matter to be determined in an absolutely noninvasive manner, thus enabling in vivo neuroanatomy in both animals and humans. If the full potential of fMRI-based DTI for tracking fiber pathways noninvasively in human and animal brains can be realized, the impact on neuroscience will be substantial. Knowing which functional areas are connected to which, and in what manner, can provide vital constraints on high-level models of global cortical organization. In short, the methods developed in this study will lay foundation for in vivo neuroanatomy and the ability for non-invasive longitudinal studies of brain development.

\section{Acknowledgments}

The authors thank Drs. Mathieu Ducros and Christoph Kayser for their helpful discussions. Supported by the National Institutes of Health (Grant PR08079), The Keck Foundation, and the Human Frontiers Science Program.

\section{References}

[1] Ogawa S, Tank DW, Menon R, Ellermann JM, Kim SG, Merkle H, Ugurbil $\mathrm{K}$. Intrinsic signal changes accompanying sensory stimulation: functional brain mapping with magnetic resonance imaging. Proc Natl Acad Sci USA 1992;89:5951-5.

[2] Bandettini PA, Wong EC, Hinks RS, Tikofsky RS, Hyde JS. Time course EPI of human brain function during task activation. Magn Reson Med 1992;25:390-7.

[3] Kwong KK, Belliveau JW, Chesler DA, et al. Dynamic magnetic resonance imaging of human brain activity during primary sensory stimulation. Proc Natl Acad Sci USA 1992;89:5675-9.

[4] Engel SA, Rumelhart DE, Wandell BA, Lee AT, Glover GH, Chichilnisky EJ, Shadlen MN. fMRI of human visual cortex. Nature 1994; 396:525.

[5] Kim SG, Ashe J, Georgopoulos AP, Merkle H, Ellermann JM, Menon RS, Ogawa S, Ugurbil K. Functional imaging of human motor cortex at high magnetic field. J Neurophysiol 1993;69:297-302.

[6] Wagner AD, Schacter DL, Rotte M, Koutstaal W, Maril A, Dale AM, Rosen BR, Buckner RL. Building memories: remembering and forgetting of verbal experiences as predicted by brain activity. Science 1998;281:1188-91.

[7] Moseley ME, Cohen Y, Mintorovitch J, Kucharczyk J, Weinstein PR. Early detection of regional cerebral ischemia: comparison of diffusion- and T2-weighted MRI and spectroscopy. Magn Reson Med 1990; $14: 330-6$.

[8] Stejskal EO, Tanner JE. Restricted self-diffusion of protons in colloidal systems by the pulse-gradient, spin-echo method. J Chem Phys 1968;49:1768-77.

[9] Mori S, Barker PB. Diffusion magnetic resonance imaging: its principle and applications. Anat Rec 1999;257:102-9.

[10] Basser PJ, Pajevic S, Pierpaoli C, Duda J, A. Aldroubi A. In vivo fiber tractography using DT-MRI data. Magn Reson Med 2000:44;625-32.

[11] Mori S, Crain BJ, Chacko VP, van Zijl PC. Three-dimensional tracking of axonal projections in the brain by magnetic resonance imaging. Ann Neurol 1999;45:265-9.

[12] Conturo TE, Lori NF, Cull TS, Akbudak E, Snyder AZ, Shimony JS, McKinstry RC, Burton H, Raichle ME. Tracking neuronal fiber pathways in the living human brain. Proc Natl Acad Sci USA 1999;96: $10422-7$. 
[13] Pajevic S, Basser P. A continuous tensor field approximation for DT-MRI data. Ninth Annul Conference of the ISMRM, 2001.

[14] Poupon B, Clark CA, Frouin V, Regis J, Bloch I, Le Bihan D, Mangin J. Regularization of diffusion-based direction maps for the tracking of brain white matter fascicles. Neuroimage 2000;12:184-95.

[15] Xue R, van Zijl PC, Crain BJ, Solaiyappan M, Mori S. In vivo three-dimensional reconstruction of rat brain axonal projections by diffusion tensor imaging. Magn Reson Med 1999;42:1123-7.

[16] Kim D-S, Ronen I, Formisano E, Ugurbil K, Mori S, Goebel R. Simultaneous, in vivo visualization of axonal connectivity and functional activity in cat visual cortex using MRI. Presented at Soc. Neurosci. Abstr. 783.3, 2001.

[17] Mori S, van Zijl PC. Diffusion weighting by the trace of the diffusion tensor within a single scan. Magn Reson Med 1995;33:41-52.

[18] Kim K-H, Ronen I, Formisano E, Mori S, Ugurbil K, Goebel R, Kim D-S. A novel fiber-tracking method using vector criterion and predictive directionality in diffusion tensor imaging. Presented at ISMSM, 2002.
[19] Kriegeskorte N, Goebel R. An efficient algorithm for topologically correct segmentation of the cortical sheet in anatomical MR volumes. Neuroimage 2001;14:329-46.

[20] Pribram K, MacLean P. Neuronographci analysis of medial and basal cerebral cortex. J Neurophysiol 1953;16:324-40.

[21] Whitlock CG, Nauta WJH. Subcortical projections from temporal neocortex in macaca mulata. J Comp Neurol 1956;106:183-212.

[22] Galuske RA, Schlote W, Bratzke H, Singer W. Interhemispheric asymmetries of the modular structure in human temporal cortex. Science 2000;289:1946-9.

[23] Katz LC, Iarovici DM. Green fluorescent latex microspheres: a new retrograde tracer. Neuroscience 1990;34:511-20.

[24] Kisvarday ZF, Kim DS, Eysel UT, Bonhoeffer T. Relationship between lateral inhibitory connections and the topography of the orientation map in cat visual cortex. Eur J Neurosci 1994;6:1619-32.

[25] Pautler RG, Silva AC, Koretsky AP. In vivo neuronal tract tracing using manganese-enhanced magnetic resonance imaging. Magn Reson Med 1998;40:740-8. 